\title{
MMW-HOTLINE
}

Leser der MMW können sich mit allen Fragen zur Abrechnung und Praxisführung an Helmut Walbert, Facharzt für Allgemeinmedizin, Würzburg, wenden. Sie erreichen ihn jeden Donnerstag von 13 bis 15 Uhr unter der kostenlosen Rufnummer (0800) 2379830 oder per E-Mail: w@lbert.info.

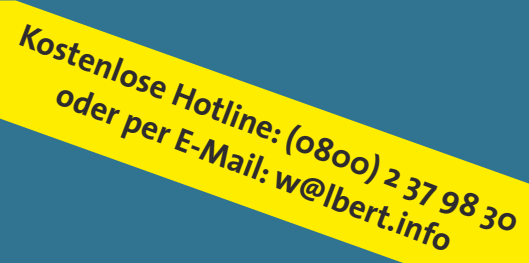

Helmut Walbert

Allgemeinarzt, Medizinjournalist und Betriebswirt Medizin

\section{Was kann ich für die Eigenbluttherapie berechnen?}

Frage von Dr. med. P. S., Allgemeinarzt, Naturheilkunde, KV Hamburg: Ich bin naturheilkundlich ausgerichtet und biete beispielsweise Eigenbluttherapie bei Psoriasis in meiner Praxis an. Wie rechne ich diese $a b$ ?

Antwort: Diese Behandlungsmethode ist als therapeutische Option zulasten der Gesetzlichen Krankenversicherung (GKV) nicht anerkannt. Aus diesem Grunde ist Eigenbluttherapie bei GKV-Patienten eindeutig eine Individuelle Gesundheitsleistung (IGeL). Aber auch privat Versicherte erhalten mittlerweile nicht immer eine Erstattung. Dies betrifft besonders Beihilfeberechtigte.

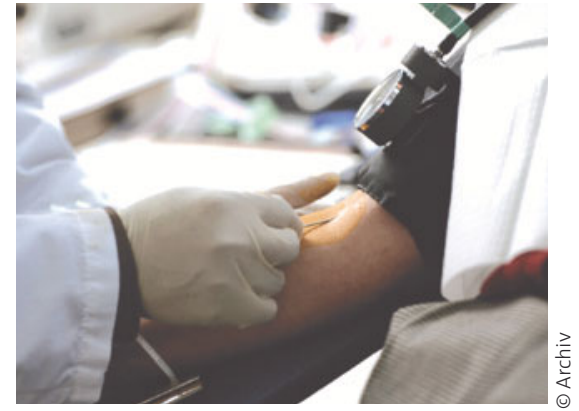

Vor einer Eigenbluttherapie Behandlungsvertrag schließen.

Beihilfestellen reagieren in letzter Zeit sehr restriktiv, weil sie den Beamten nur noch das Leistungsspektrum gewähren, das der Gesetzgeber für die GKV vorsieht.
Schließen Sie also in jedem Fall vorher einen schriftlichen Behandlungsvertrag mit allen IGeL-Patienten.

Abrechnungstechnisch kommen die Beratungsleistungen GOP 1 oder 3, je nach zeitlichem Aufwand infrage. An körperlichen Untersuchungen ist je nach Indikation GOP 5, 6 oder 7 aber auch der Ganzkörperstatus GOP 8 sinnvoll. Für die „Eigenbluteinspritzung“ gibt es in der GOÄ die GOP 284, ,Eigenbluteinspritzung - einschließlich Blutentnahme, 90 Punkte.“ Als alleinige Leistung bringt sie 2,3-fach berechnet 12,07 $€$. Eine Aufteilung in Blutentnahme GOP 250 und Injektion nach GOP 252 macht keinen Sinn, da beide zusammen nur 80 Punkte bringen.

\section{Gemeinschaftspraxis: Die Helferinnen tragen die Leistungen meist bei mir ein}

\section{Kann es da Abrechnungsprobleme geben?}

Frage von Dr. med. E.H. Facharzt für Allgemeinmedizin, München: Ich habe seit circa einem Jahr eine Gemeinschaftspraxis mit einer jungen Kollegin. Bei der Abrechnung tragen die Helferinnen die erbrachten Leistungen meist bei mir ein. Auch die meisten Rezepte laufen unter meinem Namen. Ich habe gehört, dies könne zu Problemen bei der Abrechnung und mit dem Arzneimittelbudget führen. Was ist zu beachten?
Antwort: In der Tat: Probleme sind im Rahmen der Plausibilitätsprüfung beim Tagesund Quartals-Zeitprofil zu erwarten. Aus diesem Grunde ist es seit Einführung der lebenslangen-Arztnummer (LAN) wichtig, die Leistung dem jeweiligen Erbringer zuzuordnen. Nur so kann eine Überschreitung des Tages- und Quartalsprofils bei Ihnen vermieden werden. Damit wird konsequenterweise auch eine Plausibilitätsprüfung in Bezug auf das Zeitleistungsprofil vermieden.
Bei der Analyse und Zuordnung der Verordnungen gilt, dass das Rezept demjenigen zugeordnet wird, dessen Stempelaufdruck das Rezept trägt. Auch hier ist es mehr als sinnvoll, die Verordnungen dem Arzt zuzuordnen, der sie veranlasst hat. Nur so kann im Falle einer Regressforderung im Rahmen einer Analyse der jeweilige Veranlasser festgestellt werden und die Verantwortung bzw. die Begründung übernehmen. 